Revue d'histoire de l'Amérique française

AG REVUE D.HISTOIRE DE L'AMÉRIQUE FRANÇAISE

\title{
La direction de la Nouvelle-France par le ministère de la Marine
}

\section{R. La Roque de Roquebrune}

Volume 6, numéro 4, mars 1953

URI : https://id.erudit.org/iderudit/301548ar

DOI : https://doi.org/10.7202/301548ar

Aller au sommaire du numéro

Éditeur(s)

Institut d'histoire de l'Amérique française

ISSN

0035-2357 (imprimé)

1492-1383 (numérique)

Découvrir la revue

Citer cet article

La Roque de Roquebrune, R. (1953). La direction de la Nouvelle-France par le ministère de la Marine. Revue d'histoire de l'Amérique française, 6(4), 470-488. https://doi.org/10.7202/301548ar d'utilisation que vous pouvez consulter en ligne.

https://apropos.erudit.org/fr/usagers/politique-dutilisation/ 


\section{LA DIRECTION DE LA NOUVELLE-FRANCE PAR LE MINISTERE DE LA MARINE}

Le roi de France a été un souverain absolu jusqu'à la Révolution qui a guillotiné Louis XVI. Cet absolutisme de la monarchie française a créé dans l'imagination populaire une image aussi simpliste que haute en couleur, une figure de roi qui est celle d'un tyran. En fait, aucun des quarante rois qui ont régné en France n'a été un tyran. Roi absolu, le souverain a toujours affirmé ce "droit" de la monarchie française. Dans les grandes occasions, il ne manquait jamais de le rappeler à son peuple. Le 3 mars 1766, au Parlement de Paris, dans un lit de justice, Louis XV déclarait: "C'est en ma personne seule que réside l'autorité souveraine, l'ordre public émane tout entier de moi, c'est à moi seul qu'appartient le pouvoir." Mais si Louis XV parlait ainsi à son Parlement c'est que celui-ci discutait ses ordres et refusait même de lui obéir. Car ce roi absolu que fut le roi de France eut toujours à compter avec ses Parlements, ses Etats Provinciaux, son Conseil. Et le souverain en recevait ce que l'on appelait des Remontrances. Remontrances dont il était le plus souvent forcé de tenir compte.

L'administration du royaume échappait totalement au roi qui en laissait le soin aux ministres et aux fonctionnaires des ministères. Sauf Louis XI et sauf Louis XIV qui furent des souverains bureaucrates, les Valois et les Bourbons gouvernèrent leur Etat par délégation. Et les Edits, les Ordonnances, les Lettres royaux revêtus de la signature du souverain ne portent le plus souvent qu'une imitation de l'écriture royale. Louis XIV lui-même avait un secrétaire qui arborait le titre officiel de Secrétaire de la Main et dont la charge était d'imiter sa signature. Ce secrétaire se nommait $M$. Rose. La plus grande partie des documents signés LOUIS, de l'époque de Louis XIV, sont passés par "la main" de M. Rose et le LOUIS autoritaire et royal, e'est presque toujours M. Rose qui l'a tracé.

Souverain absolu, le roi n'était pourtant pas toujours le maître. Dans les grands événements des règnes de Louis XIV, Louis XV et 
Louis XVI, quelle est la part de responsabilité du monarque? Quand on connait, quand on étudie l'entourage du prinee, on s'aperȩoit qu'il a compté pour bien peu dans les grandes décisions de son règne. Louis XIV a laissé dans l'histoire une réputation bien établie de souverain autoritaire. Et pourtant, c'est son ministre de la guerre Louvois qui lui a fait entreprendre ses funestes luttes contre l'Europe qui ont tant affaibli la France. La plus grande sottise de son règne, la Révocation de l'Édit de Nantes, c'est Mme de Maintenon qui l'a inspirée. Les ministres, les favoris, les maítresses, la reine gouvernaient le royaume par procuration. Lorsque Louis XV, en 1755-56, prend la décision de renverser les alliances de la France et signe les deux Traités de Versailles avec l'Autriche, il subissait depuis des mois l'influence de Conti, de Broglie et de Mme de Pompadour. La guerre de Sept Ans, ainsi que la défaite de Rossbach et la perte du Canada, Louis XV en porte peut-être moins la responsabilité que ses conseillers du Renversement des Alliances, origine de tous les malheurs. Quant à Louis XVI, le dernier des rois "absolus", il faut bien avouer qu'il eut la malchance de trop bien suivre les avis de son épouse, laquelle lui en donna de tout à fait désastreux, comme la nomination de Brienne ou le voyage de Varennes. De sorte que ce fut un vrai malheur pour la France que Louis XV eat été l'amant de Mme de Pompadour et Louis XVI le vertueux époux de la reine Marie-Antoinette.

A la fin de son règne, Napoléon III disait mélancoliquement: "Je n'ai aucune influence". L'empereur disait là une chose qui eût pu être pensée par tous les souverains français.

Pourtant, le roi de France travaillait. Chaque semaine, il tenait Conseil, c'est-à-dire qu'il recevait ses ministres, discutait avec eux des affaires du royaume, était mis au courant de chaque "Département" et prenait les décisions. Il est resté dans les archives de France des traces de ce "Travail du Roi". Ce sont de simples feuilles de papier sur quoi les commis (fonctionnaires) des différents ministères ont rédigé en termes très clairs et très courts un exposé d'une question à trancher par le souverain. En marge de ces documents, on lit parfois un mot, un seul, le mot bon. C'est ce que l'on appelait le Bon du Roi. La plupart des Bons du Roi sont perdus parce que ces feuilles volantes ne furent jamais reliées ensemble. On en trouve cependant quelques-uns qui se sont glissés dans des liasses ou des volumes 
de dépêches. Ces petites feuilles de papier portant le bon sont tout ce qui reste de la volonté royale.

Sous les trois derniers rois de France le Conseil se tenait au palais de Versailles, dans un salon attenant à la galerie des glaces. Les ministres, traversant la foule des courtisans qui se tenait à demeure dans cette galerie, entraient tour à tour chez le roi. Sortant de son grand portefeuille les petits papiers rédigés par les commis, le ministre recevait les instructions du souverain. Si Louis XIV admettait ses ministres plusieurs fois par semaine, Louis $\mathrm{XV}$ que le travail du roi ennuyait, ne les voyait que très rarement. Les très grandes questions étaient donc les seules qui eussent chance de passer sous les yeux du chef de l'Etat. Tout ce qui tenait à ce que l'on appelait "le détail "était du ressort des ministres, des intendants dans les provinces et surtout des "Commis". Il ne pouvait en être autrement dans un royaume qui avait dix-neuf millions d'habitants au XVIIe siècle et vingt-cinq millions au XVIIIe. Mais, dans ces conditions, il faut bien avouer que l'absolutisme du roi de France ne s'exerçait pas souvent.

C'est pourquoi on a pu dire très justement qu'en France le roi faisait administrer son royaume comme un grand seigneur faisait administrer ses domaines: par les fonctionnaires ${ }^{1}$. Et comme le Canada a fait partie du royaume de France pendant tout le règne de Louis XIV et une partie de celui de Louis XV, il n'est peut-être pas sans intérêt de savoir qui étaient ces fonctionnaires, ce qu'ils étaient surtout et quelle part ils ont eue dans l'administration de la colonie. Le Canada ayant toujours été du ressort du ministère de la Marine, c'est donc ce ministère et ses fonctionnaires que nous voulons surtout étudier ici.

Le ministère ou, ainsi qu'on le nommait alors, le Département de la Marine a réellement commencé au XVIe siècle avec la nomination en 1547 de Clausse de Marchaumont qui reçut le titre de Secrétaire d'Etat à la Marine. La Marine fut ainsi l'un des premiers ministères régulièrement constitués puisque celui des Affaires Etran.

1. G. Dagnaud, L'Administration centrale de la Marine sous l'ancien régime (Naney). 
gères ne date en France que de 1589 avec la nomination de Revol au poste de Secrétaire d'Etat. La Marine releva d'abord de l'Amiral de France. Mais Henri de Montmorency ayant pris part à l'essai de soulèvement de Gaston d'Orléans contre le cardinal de Richelieu, fut décapité à Toulouse. Ni sa charge d'amiral de France ni la grande situation de sa famille n'avaient pu le sauver. Parmi ses nombreux titres Montmorency avait celui de vice-roi de la Nouvelle-France. Après la mort de l'amiral le cardinal ministre dirigea la marine et les colonies avec le titre de Grand Maitre, Chef et Surintendant de la Navigation et du Commerce. Potier d'Ocquerre assurait l'administration du Ponant, Le Beaucle d'Achères celle du Levant. Tous deux sont les plus anciens commis de la Marine ${ }^{2}$.

Après la mort du cardinal, la charge d'Amiral de France fut rétablie en faveur d'un prince du sang, le duc de Beaufort. Mais c'est Lyonne qui signait et qui donnait les ordres, car Beaufort était rien moins qu'un bureaucrate. La direction effective de la Marine et des Colonies était aux mains des commis, car Lyonne étant Secrétaire d'Etat aux Affaires Etrangères n'entendait rien à la marine. Beaufort encore moins quoiqu'il prétendît s'en mêler en vertu de sa charge. Jadis, la régente Anne d'Autriche n'avait-elle pas exercé cette charge avec le titre de Grande Maîtresse de la Navigation! C'est pourquoi tout se faisait par le commis. Leur règne date vraiment de la minorité de Louis XIV.

Il est impossible de retrouver leurs noms car ils n'ont pas laissé trace de leur passage. C'est seulement avec Colbert que les fonctionnaires de la Marine commencent à avoir une figure et que l'on peut mettre un patronyme sur leurs silhouettes apparues dans l'ombre $\mathrm{du}$ ministre.

Colbert commença à s'occuper de la marine dès 1665 car il voulait "la rendre à la monarchie"3. En effet, depuis longtemps, tous les droits souverains: nomination des officiers, entretien de la flotte etc., appartenaient au Grand Amiral. La mort du duc de Beaufort, tué au siège de Candie, délivra Colbert d'une tutelle gênante. Afin de n'avoir plus à compter avec quelque prince stupide, comme l'avait été l'héroïque Beaufort, le rusé Colbert persuada Louis XIV de

2. G. Dagnaud. op. cit.

3. Charles de La Roncière, Histoire de la Marine française (Paris, 1920). 
faire Amiral de France le duc de Vermandois. Avec ce prince, Colbert n'avait pas à redouter d'ennuis, car c'était un enfant de deux ans. Bâtard de Louis XIV et de Mlle de La Vallière, bébé Vermandois eut les mêmes titres que Beaufort. La nomination de l'amiral enfant, "capitaine général de la mer," est un coup de Colbert pour s'emparer de la Marine, écrivait l'ambassadeur du duc de Savoie à son maîtré. En 1669, le roi créait la charge de Secrétaire d'État à la Marine et c'est Colbert qui en devenait le titulaire. Ce fut l'origine du Ministère de la Marine. Désormais il y aura un Amiral de France mais dont les attributions et les pouvoirs seront tout à fait limités et sans action sur le ministère, lequel sera tout entier entre les mains $\mathrm{du}$ ministre et de ses commis.

Colbert en eut d'abord trois. On ne connalt que Clairambault, que l'on appelait Clairambault l'aîné ou Clairambault l'oncle pour le distinguer d'un neveu nanti d'une charge de cour, celle de généalogiste. Clairambault est signalé commis "de M. Colbert" en 1663 et "commis des fonds" en $1678^{5}$. Colbert assumant la direction non seulement de la marine mais aussi du commerce, des consulats et des colonies, dut s'adjoindre bientôt plusieurs fonctionnaires. En 1683 , à sa mort, il en avait une dizaine dans ses bureaux. Ces "comis", car telle est l'orthographe ancienne de ce mot, étaient Le Foin, Desmarets fils, Roze de Coye, Clairambault, Bessard, Charles Perrault, de Matucières, de La Grange et "M. de La Garde" qui paraît avoir exercé les fonctions de Premier Commis sans en avoir réellement le titre. La Garde avait parfois la signature des dépêches ${ }^{6}$.

$\mathrm{Au}$ décès de Colbert, son fils, le marquis de Seignelay, lui succède dans sa charge. Depuis dix ans Seignelay travaillait "sous Monsieur son père" avec qui il avait "appris la Marine". Ambitieux et intelligent, le jeune ministre voulait que la marine de France fat très forte et que les colonies fussent développées. La mort ne lui laissa pas le temps d'accomplir ses grands desseins. Il mourut à trente-neuf ans en 1690 et fut remplacé par le comte de Pontchartrain.

4. Marquis de St-Maurice, Lettres sur la cour de Louis XIV, publíés par Jean Lemoine (Paris, 1911), 363.

5. AM., [Archives de la Marine] C 2, 55.

6. Registres du Secrétariat d'Etat, B.n. F.F. 2771-72. 
Avec Seignelay commence le règne des Premiers Commis qui deviennent des personnages importants. Ils deviendront plus tard, sous les ministres de Louis XV, des personnages considérables. Déjà d'Usson de Bonrepaus avait la situation de Secrétaire d'Etat auprès du marquis de Seignelay et, à la mort de ce dernier, on crut qu'il lui succéderait. Le roi nomma Louis Phélypeaux de Pontchartrain, mais M. de Bonrepaus devint Lieutenant Général des Armées Navales. Sous le ministère Seignelay les deux Premiers Commis sont MM. de Valocières et de Morel-Boistiroux. Le poste est désormais occupé par de grands fonctionnaires qui ont un rang élevé dans l'administration de la Marine, du Commerce et des Colonies.

Avec Pontchartrain le ministère est dirigé par deux Premiers Commis: M. de La Touche pour le Ponant, M. d'Irumberry de Salaberry pour le Levant. De La Touche relevaient les ports de Bordeaux, Rochefort, Brest, Dunkerque, le Havre. Le commerce, les compagnies, le Canada, les armements, la construction des navires, les achats, les munitions, inspections et nominations des officiers étaient de son ressort. De Salaberry relevaient les ports de Toulon et Marseille, le commerce du Levant, Alger, Tunis, les consulats "aux Barbaresques", d'Italie, d'Espagne, enfin "les îles d'Amérique". Sous La Touche et Salaberry travaillent quatre commis qui sont MM. de Silvecanne, Le Vasseur, de La Coudrais et Clairambault jeune. Un bureau "des fonds" fut bientôt adjoint à l'administration centrale avec M. Begon comme commis et un bureau du commerce dirigé par M. de Lagny. Le nombre des commis augmenta sans cesse avec l'importance des bureaux de la Marine. En 1715, on trouve quatre Premiers Commis et en 1729 cinq avec neuf seconds commis chargés "des détails". En 1740, il y a huit Premiers Commis à la Marine et un nombre croissant de seconds, troisièmes commis et secrétaires. Au milieu du XVIIIe siècle, le ministère se composait de huit bureaux: Fonds, classes, comptes, police des ports, commerce et consulats, les colonies, officiers d'épée et de plume, dépôt des cartes et plans, secrétariat. En 1753 , le personnel comptait soixantecinq commis. Certains premiers commis demeurèrent en poste très longtemps, ce qui leur assurait ${ }^{7}$ une véritable dictature sur toute 1886).

7. H. de Resbecq, L'Administration centrale de la Marine et des Colonies (Paris, 
l'administration de la Marine et des Colonies. M. de Fontanieu fut Premier Commis de 1710 à 1725, M. de Forcade le fut de 1725 à 1738, M. Arnaud de La Porte de 1738 à 1758, M. Accaron de 1758 à 1764 seulement et M. Dubuc de 1764 à 1770, mais MM. de La Coste et de La Rivière de 1770 à la Révolution.

Le titre de commis qui paraît modeste aujourd'hui, ne l'était nullement dans la langue de l'administration de l'Ancien Régime. Commis équivaut au civil servant que les Anglais ont conservé pour désigner les fonctionnaires. Les traitements des commis de la Marine étaient plus élevés que ceux des officiers de la Marine Royale. De même leurs pensions. En $1758 \mathrm{M}$. de La Porte est mis à la retraite avec 13.000 livres de pension pour vingt huit ans de service. En 1760 M. Chevalier a 8.000 livres de pension pour cinq ans de service. En 1760 M. Le Tourneur qui a quarante ans de service obtient une retraite de 24.000 livres; et $M$. Accaron qui a servi vingt ans reçoit 24.000 livres. M. de La Roque de Couillaud pour ses vingt-huit ans de service a une pension de 11.000 livres en 1774 et $M$. de Rosières pour deux années de service a 4000 livres $^{8}$. A la même époque les capitaines de vaisseau n'obtenaient que 3000 et 4000 livres de pension pour les plus longs services. Il était beaucoup plus lucratif de servir dans les bureaux de la Marine que sur les navires du Roi.

Les commis de la Marine se recrutaient dans la noblesse et la haute bourgeoisie. Le poste n'étant pas une charge n'était pas anoblissant d'office, mais le roi accordait des Lettres d'anoblissement aux commis qui n'étaient pas nobles. Cette mesure concernait tous les commis, qu'ils fussent du Département de la Marine, de la Guerre ou des Affaires Etrangères. Peu à peu d'ailleurs la noblesse et les militaires pénétrèrent dans les bureaux de la Marine".

Certains Premiers Commis furent les véritables maîtres de leur Département et de leur ministre. Fonctionnaires rompus au métier, ils conduisaient tout, inspiraient les décisions et même les prenaient à l'insu du ministre et du roi. Arnaud de La Porte à la Marine, Ticquet aux Affaires Etrangères, furent des dictateurs. Le ministre d'Argenson prétendait qu'aux Affaires Etrangères "Ticquet mène

8. AM., C 2, 121.

9. G. Dagnaud, op. cit. 
tout"10. A la guerre le Premier Commis Bricquet était, disait-on, le ministre sans le titre. La situation des premiers commis leur donnait un prestige énorme. "Tous ces grands commis de l'ancien régime, La Porte, Forcade, Dubuc pour n'en citer que quelques-uns, étaient des hommes d'importance. Du fait de leurs fonctions, ils avaient un rôle officiel, une charge publique. Ils étaient souvent des sousministres ${ }^{11}$."

Les Premiers Commis avaient entre les mains la direction de la politique du royaume aux Affaires Etrangères, à la Guerre, à la Marine. Ils tenaient "tous les fils de la politique coloniale"12. Nul ne s'interpose entre les Premiers Commis et le ministre qui parfois les met en relation directe avec le ro ${ }^{13}$. Pecquet, Premier Commis aux Affaires Etrangères, travaillait intimement avec Louis XV, lequel s'amusait à contrecarrer la politique étrangère de son ministre le cardinal de Fleury. Le roi trouvait comique de "faire la barbe au cardinal" avec l'aide de M. Pecquet ${ }^{14}$. Fleury finit par découvrir l'intrigue royale. Il ordonna l'arrestation de Pecquet et Louis XV n'osa defendre son complice. Mis à la Bastille, Pecquet y demeura "par ordre du Roi" et ses papiers furent saisis ${ }^{15}$. "Avec son caractère cauteleux et faible, le souverain ne songea pas à défendre le commis qui n'avait fait que suivre ses instructions"'16. D'ailleurs, Louis XV avait l'habitude de ces fourberies et il était très dangereux parfois de lui obéir. Tercier, autre commis des Affaires Etrangères, en sut quelque chose. Le roi qui continua sous tous ses ministres son fâcheux système de politique secrète, se servait de Tercier pour travailler contre Choiseul. Lorsque le ministre s'en aperçut, Tercier fut sacrifié comme l'avait été Pecquet ${ }^{17}$. Au commis Favier il arriva une aven-

10. Camille Piccioni, Les premiers commis des Affaires Etrangères au XVIIe et au XंVIIIe siecle (Paris, 1905).

11. A. Duchêne, La politique coloniale de la France. Le ministère des Colonies après Richelieu (Paris).

12. ibid.

13. $i b i d$.

14. C. Piccioni, op. cit.

15. ibid.

16. ibid.

17. C. Piccioni, op. cit. 
ture semblable et pour une cause encore plus injuste. Sur l'ordre du duc de Choiseul il rédigea pour le roi un Mémoire sur la politique étrangère. Il concluait que le Renversement des Alliances en 1756 avait été néfaste pour la France. Louis XV, furieux de voir sa politique personnelle condamnée par Favier, le fit mettre à la Bastille où il resta deux ans. Ce Tableau de l'Europe par Favier est "un chef-d'œuvre de science politique"18. Favier conseillait de bloquer l'Angleterre dans son île et de détruire l'Autriche ${ }^{10}$.

Mais les premiers commis étaient en général très écoutés. Aux Affaires Etrangères, M. Le Dran fut un inspirateur constant des ministres et du roi par ses Mémoires historiques et diplomatiques. A la Marine, le commis Raudot fils inspira la politique coloniale de Louis XIV et de Pontchartrain. C'est lui qui indiqua au roi l'importance stratégique de l'île Royale pour la défense du Canada ${ }^{20}$. Lorsque le ministre était un incapable, les commis conduisaient tout. Le second Pontchartrain, Jérôme, se laissa conduire par Fontanieu. Pendant vingt ans Arnaud de La Porte fit la pluie et le beau temps à la Marine et aux Colonies. Ce qui faisait la force de ces fonctionnaires, est qu'ils demeuraient alors que les ministres changeaient. Lorsque le Régent remplaça le ministre de la Marine en 1715 par un Conseil de Marine, sous la direction du comte de Toulouse et du maréchal d'Estrées, le premier commis Fontanieu demeura en fonctions. La marine était en ruine, les colonies négligées. Le comte de Toulouse fit de dures critiques de M. de Pontchartrain qui tenta de se justifier par le manque d'argent ${ }^{21}$. Jérôme de Pontchartrain "fut réduit, a dit Saint-Simon, au mouchage des chandelles du Conseil de Marine". Mais Fontanieu resta Premier Commis jusqu'en 1725. Sous le comte de Maurepas qui fut ministre de la Marine pendant vingt six ans, les commis Forcade et Arnaud de La Porte eurent "les mains à tout". Maurepas était frivole, sans expérience de la marine et des colonies" et obligé de se livrer pour les détails à ses bureaux ${ }^{22}$."

18. ibid.

19. Le Mémoire de Favier est conservê aux Archives du Ministère des Affaires Etrangères, F.F. 583.

20. H. de Resbecq, op. cit.

21. AM, G 126.

22. AM, G 75 . 
La haute situation des commis était encore rehaussée par la noblesse des uns, la fortune considérable des autres, l'importance des familles dont ils étaient. La Porte du Theil aux Affaires Etrangères prouvait une noblesse qui remontait au XVIe siècle; Salaberry à la Marine prouvait depuis le XIVe siècle. Gourdan, commis des Affaires Etrangères était puissamment riche par sa famille. En 1723, Mme Gourdan avait un salon très couru à Versailles et la reine disait: "Je n'ai personne chez moi quand Mme Gourdan reçoit" ${ }^{23}$. En 1717, le commis de la Marine, archiviste du Dépôt des Cartes et Plans, était le chevalier de Luynes, cadet de famille ducale. Par contre, beaucoup de commis sortaient de la bourgeoisie. Le Dran était fils d'un maître-chirurgien de Paris. Le commis de la Marine Joseph Pellerin, né à Marly en 1683, était fils d'un trompette du Roi.

Les commis succédaient parfois à leur père, à leurs oncles. Il y eut des dynasties de commis comme il y avait des dynasties de ministres. Trois Phélypeaux furent ministres de la Marine (deux Pontchartrain et un Maurepas). Deux Clairambault furent commis de la Marine, deux Pellerin, trois La Porte. Les commis devaient souvent leur place à un autre commis, leur protecteur. Les familles des commis se mêlaient par des mariages. Henri de Besset de La Chapelle, fils d'un commis de la Guerre, fut secrétaire du Conseil de Marine sous la Régence. Son fils fut commis à la Marine. Arnaud de La Porte entra à la Marine par le protection de Pellerin dont il épousa la fille en $1736^{24}$.

La carrière de certains commis fut magnifique. Aux Affaires Etrangères, l'abbé de la Ville de Mirmont devint ministre plénipotentiaire, La Porte du Theil ambassadeur. A la Marine la carrière la plus célèbre est celle des Phélypeaux. Elle débuta par un Pontchartrain, commis des Affaires Etrangères sous le ministre Villeroy, et se continua par les Phélypeaux de Pontchartrain, d'Herbault et de Maurepas, chanceliers de France, ministres, présidents de Parlement. Ils étaient d'ailleurs de modeste origine, sortant d'une famille de paysans du Blésois. Salaberry devint Président de la Chambre des comptes et son fils et son petit-fils le furent après lui. Un autre de ses fils, Salaberry de Benneville, fut vice-amiral. Le fils d'Arnaud de

23. G. Dagnaud, op. cit.

24. AM, C 7. Dossier La Porte. 
La Porte fut intendant général de la Marine en 1787 et ministre de la Marine en 1789. Poste dangereux à une telle date car il fut guillotiné en 1792.

Certains commis se rendirent coupables de malversations et, pire, de trahison. M. de Bussy, commis des Affaires Etrangères, vendait des documents à l'Angleterre. Seul le marquis d'Argenson s'en doutait. "Bussy vend la France aux Anglais", disait-il. Mais personne ne voulait croire Argenson qui avait une réputation de bêtise bien établie par la Pompadour à qui son honnêteté déplaisait. Dans l'entourage de celle que le bon $M$. de Nolhac appelle respectueusement la Marquise dans les aimables et légers ouvrages qu'il lui a consacrés, on appelait "d'Argenson la bête" l'homme clairvoyant qui gênait beaucoup leurs intrigues. Mais d'Argenson la bête disait vrai. Bussy trahissait et vendait les secrets diplomatiques de la France ${ }^{25}$. Le premier commis de la Marine Arnaud de La Porte se servait de sa place pour faire des affaires. Il en faisait surtout dans les colonies dont les intendants étaient à sa dévotion. Il protégea Bigot, intendant au Canada, s'associa même avec lui et empêcha toujours les dénonciations de parvenir jusqu'au ministre ${ }^{26}$.

Mais les commis étaient en général d'honnêtes gens et beaucoup étaient des hommes de grande valeur. Aux Affaires Etrangères, Le Dran, La Ville de Mirmont, La Porte du Theil, Favier, Tourmont, Pachau furent de consommés diplomates. A la Marine, Lagny, Raudot, Pellerin, Forcade, Begon étaient des fonctionnaires intelligents et dévoués. Arnaud de La Porte, en dépit de ses malversations, fut un Premier Commis merveilleusement habile et actif. Il dirigea supérieurement le ministère de la Marine pendant deux guerres: celle de la succession d'Autriche et la Guerre de Sept $\mathrm{Ans}^{27}$. On put apprécier La Porte lorsque Accaron prit sa place. Il ne connaissait rien aux colonies. "Dépassé par les événements pendant la guerre... il subit le traité de Paris de 1763 sans essayer de défendre les colonies. Il n'avait pu ou su rien empêcher" ${ }^{28}$. Choiseul se débarrassa

25. C. Piccioni, op. cit.

26. Guy Frégault, François Bigot (2 vol., Montréal, 1948) I: 290.

27. A. Duchêne, op. cit.

28. ibid. 
de lui. "Mais le prestige qui entourait le titre de Premier Commis lui fit marier sa fille au comte de Grasse" 29 . Car les "commis", enviés et redoutés, étaient de hauts personnages.

Ces commis de tant de puissance dans l'Etat demeuraient tous à Versailles, près du roi et des ministres. L'hôtel des Affaires Etrangères existe toujours dans la cité royale. C'est aujourd'hui la Bibliothèque de la ville. Le ministère de la Marine et des Colonies était, à Versailles, rue des Bons Enfants. C'est dans cette maison que travaillèrent les commis de la Marine, c'est de là que partirent tant de dépêches, tant de Mémoires pour le Canada, c'est là qu'arrivaient les lettres des gouverneurs et intendants de la Nouvelle-France marquées en "Cour". C'est dans cette maison de la rue des Bons Enfants que les commis de la Marine rédigèrent les Feuilles au Ministre et les Feuilles au Roi qui résumaient les dépêches et permettaient à Seignelay, à Pontchartrain, au comte de Toulouse, à Maurepas, à Berryer, à Choiseul, de se présenter devant Louis XIV et devant Louis XV et de les mettre au courant des affaires concernant la colonie.

Si on se penche sur les documents qui forment aux Archives des Colonies la série CII., on aperçoit dans les marges des lettres venues du Canada, des notes, des apostilles mystérieuses. Mystérieuses seulement pour qui ne connait pas la manière de travailler des commis de la Marine. Ces apostilles sont de courts résumés des dépêches par un commis. Souvent une note est d'un ton péremptoire, c'est un ordre en quelques lignes, une décision en trois ou quatre mots. On y reconnaît la Main du Premier Commis. On peut être assuré que cette note sera développée en une lettre rédigée par un commis et adressée au gouverneur ou à l'intendant du Canada, lettre dont la copie existe toujours dans les gros registres de la série Colonies B. des Archives, lettres dont les originaux sont perdus. Ces lettres-là ne passaient jamais sous les yeux du ministre, encore moins sous ceux du roi. Le Premier Commis en prenait seul la responsabilité. De sorte que la Nouvelle-France a été dirigée de Versailles, du moins en ce qui concerne les questions secondaires, par les commis de la Marine.

Sous Colbert il n'en était pas ainsi. Le grand ministre voyait tout et la correspondance avec le Canada n'échappait nullement à

29. ibid. 
sa vigilance. Dans les marges des lettres de Talon, notamment, on reconnaît la petite écriture de Colbert, son affreuse petite écriture indéchiffrable. (Colonies CII A,2. ff. 322-25. - 3 ff. 49-53) Parfois des brouillons de lettres, des Mémoires entiers sont de la main de Colbert $^{30}$. Il arrive aussi qu'un seul bon figure dans la marge. C'est le "bon" du ministre, car on reconnaît sa griffe ${ }^{31}$. Un mémoire pour l'entretien du Fort Frontenac, par La Salle en 1674, porte au revers un résumé de la main d'un fonctionnaire de la Marine. On peut lire une note en petite écriture de chat, à la suite du résumé. C'est du Colbert $^{32}$. Certains extraits ou résumés des lettres de Frontenac par un commis de la Marine portent en marge "vu" orthographié veu ou "hic". C'est de la main de Colbert qui indique qu'il a lu le document ou qu'il a pris note d'un passage particulièrement intéressant ${ }^{33}$. Enfin lorsqu'une pièce est marquée de la main de Colbert d'un "CI", cela signifie que le ministre apportera ce document chez le Roi, au Conseil ${ }^{34}$.

Ce travail sur la correspondance du Canada, Colbert eût voulu que son fils Seignelay s'y astreignit. Tant que vécut le grand ministre, on trouve des traces du labeur du jeune homme qui allait lui succéder. Dans la marge d'une dépêche de Frontenac du 16 février 1674, on lit: "à voir par mon père" et à côté: "à mon fils". C'est Colbert et Seignelay qui ont échangé la lettre du gouverneur du Canada et l'ont apostillée ${ }^{35}$. Après la mort de Colbert, les lettres venues du Canada sont encore marquées parfois dans les marges d'un "vu" ou d'un "hic" ou de notes de la main de Seignelay36. Mais, peu à peu l'écriture du jeune ministre disparaît pour faire place à celle de l'un des commis de la Marine. "Seignelay aimait la cour plus que les bureaux de la Marine" ${ }^{37}$. Il avait pourtant de grandes idées, fit

30. AC, C II A, 3: ff. 8-9.

31. AC, C II A, 4: f. 44.

32. AC, C II A, 5: ff. 97-99.

33. AC, C II A, 5: ff. 18-19.

34. AC, C II A, 5: ff. 137.

35. AC, C II 1, 4: ff. 49-54.

36. AC, C II A, 6: ff. 159 et 393.

37. G. Dagnaud, op. cit. 
beaucoup pour la Marine, voulait poursuivre les vues de son père sur le Canada. Mais la vie mondaine l'empêchait de travailler. Jadis Colbert avait écrit à son intention un grand Mémoire où il lui enseignait sa méthode de travail. "Je mettrai un mot de ma main sur chaque article de l'extrait (préparé par un commis) contenant la réponse qu'il faudra faire. Aussitôt il faudra que mon fils fasse les réponses de sa main sur chaque article de l'extrait's8. Seignelay suivit un peu les conseils de son illustre père, puis les oublia et laissa les commis non seulement préparer les extraits, mais écrire les lettres qu'il se contentait de signer.

Le grand Colbert était mort désespéré en 1683, car il avait presque perdu la confiance de Louis XIV qui ne suivait plus ses idées, mais celles de Louvois. Le roi était entraîné par Louvois dans la poursuite de la grandeur et de la gloire par la guerre. Colbert avait longtemps persuadé Louis XIV que sa gloire était de rendre le peuple français puissant par son commerce et ses colonies. Vaincu dans sa lutte contre Louvois, Colbert ne fut jamais remplacé et le colbertisme, c'est-à-dire la politique coloniale du grand ministre, ne fut plus qu'un souvenir. Le Canada sera désormais conduit de haut par un monarque distrait, par des ministres indifférents. La Colonie recevra surtout la direction des Commis.

C'est ce que l'on voit en lisant les apostilles et les notes en marge de la correspondance de la série CII. Mêlés aux lettres des gouverneurs, intendants et autres fonctionnaires de la Nouvelle-France, les Résumés et les Extraits des seconds et des troisièmes commis du ministère de la Marine sont couverts des apostilles et des notes des Premiers Commis. Ces apostilles sont presque toujours à l'encre, parfois au crayon. Dans ce cas elles sont à demi effacées par le temps mais encore déchiffrables ${ }^{39}$. C'est dans ces apostilles qu'il faut chercher à comprendre comment le Canada fut dirigé de Versailles, c'est-à-dire le plus souvent par les Commis du Département de la Marine.

38. Mémoire pour mon fils, par Colbert, 1673, cité par G. Dagnaud, op. cit.

39. Sur beaucoup de documents de la série C II, aux Archives nationales à Paris, on lit, inscrits au crayon, les noms de Marmette, Faribault ou Parkman. C'est un souvenir du sans-gêne des copistes de ces deux archivistes canadiens et de cet historien américain qui marquaient ainsi les pièces qu'on leur donnait à transcrire. 
Les deux Pontchartrain suivirent d'assez près les affaires de la Nouvelle-France si on en juge d'après les apostilles de leurs mains en marge des Résumés des commis de la Marine. Le comte de Toulouse également. Mais Maurepas et les ministres de Louis XV semblent avoir abandonné la colonie à la direction des bureaux, particulièrement aux Premiers Commis Forcade et Arnaud de La Porte.

Une lettre de Callières datée de Montréal le 20 septembre 1692 est suivie d'un Résumé de la main d'un commis de la Marine ${ }^{40}$. En marge, on lit le "bon" de la main de Pontchartrain. Sur un Mémoire du Fermier du Bail de Pointeau, daté de Québec, 4 novembre 1693, Pontchartrain a répondu en marge ${ }^{41}$. Villebon écrit à Pontchartrain du Fort de Naxouat le ler octobre 1695 et sa lettre porte en marge, de la main du ministre, "bon" et "néant"42. Toutes les lettres et dépêches venues du Canada sont adressées à "Monseigneur", c'est-àdire au ministre, mais ce sont les commis de la Marine qui y répondent en s'inspirant des directives portées en marge des lettres et des Résumés. On voit très clairement comment fonctionne le système en lisant le Résumé des dépêches de Vaudreuil en 1711, notamment. La marquise de Vaudreuil est en France depuis 1709 où "elle déploie une étonnante activité" en faveur de son époux ${ }^{43}$. On peut croire que cette intelligente personne, si elle sollicite le ministre Jérôme de Pontchartrain, ne néglige pas le Premier Commis Fontanieu. Elle finira par obtenir, grâce à la duchesse de St-Simon, la place de sous-gouvernante des enfants du duc de Berry ${ }^{44}$. Mais que de voyages à Versailles dut entreprendre Mme de Vaudreuil, que de stations dans l'antichambre du Premier Commis Fontanieu! Combien de fois se présenta-t-elle à l'audience du ministre Pontchartrain? Sur un Résumé d'une lettre de Vaudreuil datée de Québec en 1711, on lit: "Il marque qu'ayant appris que les ennemis le devaient attaquer, il a fait descendre tous les sauvages d'en haut"... En marge, Pontchartrain a écrit au crayon: "Bon, louer". "Il assure, continue le Résumé

40. AC, C II A. 12: f. 100.

41. AC, C II A. 12: ff. 291-93.

42. AC, C II D. 2: ff. 277-78.

43. Guy Frégault, Le grand marquis (Montréal, 1952), 73.

44. Mémoires de Saint-Simon (Ed. Boislisle, Paris, 1911), XXIII: 221; voir aussi Francis H. Hammang, The Marquis de Vaudreuil (Louvain, 1938). 
du commis, qu'il donne toute son attention"... En marge, le ministre écrit: "Bon, ne peut trop". "Il supplie, résume le commis, il supplie Sa Majesté de le faire commandeur de l'ordre de St-Louis..." En marge: "Bon, sans pension et bien marquer dans les expéditions que c'est sans pension ni espérances. Me parler la prochaine fois". "Et de lui accorder gratification, continue le commis de la Marine, ou une pension pour lui et son épouse qui la puisse faire vivre avec ses enfants, dont quatre dans le service et qui se trouvent sans aucun bien". En regard de cette dernière demande, le ministre a écrit: "Le Roy y aura attention, il peut être tranquille sur cela." Ces apostilles, de la grosse écriture bien caractéristique de Jérôme de Pontchartrain, au crayon et presqu'effacées, ont été copiées à l'encre par un commis ${ }^{45}$. Et, au dos de ce Résumé, le Premier Commis Fontanieu a écrit de sa fine écriture pressée et rapide: "Suivre les apostilles. Faites, je vous prie, mes compliments à Mme de Vaudreuil de ce que je lui ai fait obtenir. Faites lui entendre que j'ai eu bien de la peine à obtenir ce que j'ai obtenu pour lui" ${ }^{46}$. Suivant avec soin les instructions des apostilles du ministre et de la note du Premier Commis, un commis de la Marine a rédigé une lettre pour le marquis de Vaudreuil, lettre dont l'original est perdu mais heureusement demeurée en copie dans les gros Registres de Colonies B.

Lorsque le Régent Philippe d'Orléans supprima les Ministères en 1715 et les remplaça par des Conseils, le comte de Toulouse dirigea celui de la Marine. Le Conseil de Marine se réunissait au Louvre. Il comprenait Louis-Alexandre de Bourbon, comte de Toulouse, Chef du Conseil, le maréchal d'Estrées, président, l'amiral de Cœtlogon, Antoine Ferrand, Maître des Requêtes, le chef d'escadre Bouchard de Champigny, le chef d'escadre Girardin de Vauvré et le chef d'escadre Elisée de Cours. Le secrétaire du Conseil de Marine était le commis Besset de La Chapelle. Les délibérations du Conseil de Marine étaient signées L.A. de Bourbon et Amiral d'Estrées et contresignées La Chapelle. Sur les Résumés ou Feuilles au Conseil

45. Ce document se trouve dans Colonies C II A, vol. 32, ff. 156-61. Si on compare les apostilles, au crayon, de cette pièce, avec une lettre de la main de Jérôme de Pontchartrain, on reconnaît l'identité des écritures. Voir: Archives nationales, Paris, Documents originaux, Musée des Archives nationales (pub. par la Direction des Archives, 1872), un fac-similé de l'écriture de Jérôme de Pontchartrain, p. 547.

46. AC, C II A. 32: ff. 156-61. 
de Marine préparés par les commis d'après la correspondance du Canada, on trouve souvent des apostilles de la main du comte de Toulouse. Ces notes portent un "A" ce qui signifie Alexandre. Parfois les notes en marge sont signées L.A.B. et L.M.D. ce qui veut dire Louis-Alexandre de Bourbon et Le Maréchal d'Estrées ${ }^{47}$.

A la suppression du Conseil de Marine en 1723, la NouvelleFrance est de nouveau sous la direction du ministre, mais les Premiers Commis dirigent tout "le détail". Et le détail est bien souvent l'essentiel. De 1725 à 1758 deux hommes ont occupé le poste de premier commis à la Marine: Forcade et Arnaud de La Porte. On peut dire que le Canada a été sous la coupe de ces deux fonctionnaires pendant tout le règne de Louis XV. La Porte surtout a joué dans l'histoire du Canada un rôle immense, totalement ignoré des historiens et d'ailleurs bien difficile à pénétrer. Sur toutes les lettres venues de la Nouvelle-France, on trouve, de la main d'un commis, à l'encre ou au crayon, la mention: "M. de La Porte" ou simplement: "M. de La P." Ce qui signifie que La Porte lira la lettre et y fera faire réponse $^{48}$. Et on peut penser que le ministre n'a pas toujours pris la peine de lire les lettres pour la colonie que l'on portait à sa signature. Il en était de même dans tous les ministères. Le duc de Choiseul disait que "les commis barbouillaient les notes et que le ministre n'y mettait que les virgules"

"Les commis laborieux et instruits, sous le nom des ministres, dirigeaient l'administration française" a dit Frédéric Masson et le duc de Broglie leur a rendu justice lorsqu'il écrivait: "Le poste de premier commis dans notre ancienne organisation était très important. Survivant aux ministres, dépositaires des traditions, les premiers commis ont été vraiment pendant des siècles, du fond de leurs cabinets, le ressort fixe et principal, bien qu'inaperçu de toute notre politique extérieure" ${ }^{150}$. Le roi et les ministres avaient pour les commis

47. AC, C II D, 10: Volume non folioté. 4 Janvier 1716: Feuille au Conseil de Marine. Délibération signée L.A. de Bourbon, Le Maréchal D'Estrées. Contresignée La Chapelle. - La décision est en marge signée L.A.B. et L.M.D. - Voir aussi C II B, I. ff. 457-59, en marge d'une lettre de Brouillan une note de la main du comte de Toulouse signée " $A$ ".

48. AC, C II B, 33, f. 54. - C II B, 34. f. 247.

49. Baschet, Histoire du Dépót des Archives des Affaires Etrangères, 440.

50. Duc de Broglie, Le secret du Roi. 
la plus haute considération, car les services de ces fonctionnaires leur étaient indispensables et précieux. "La monarchie avait pour ses premiers commis des trésors d'indulgence. On ne cessa d'accroître leurs traitements et leurs pensions. Le Service du Roi méritait tout cela"'51. Les commis abusaient parfois de leur pouvoir et se servaient de leur place pour faire fortune. A la Marine, Arnaud de La Porte est resté l'exemple le plus connu parmi les fonctionnaires coupables de malversations. Il finit par être renvoyé mais avec une bonne pension. La construction des navires, les armements, les vivres et les fournitures de toutes sortes donnaient aux premiers commis trop de facilités pour que certains d'entre eux ne fussent pas tentés d'en profiter. Dans un Mémoire anonyme mais qui semble bien provenir d'un officier de la Marine royale, on trouve ces réflexions amères: "L'Etat ne peut plus payer ses dettes, les équipages ne sont plus soldés. Le Roi est volé, la destination de ses fonds n'est jamais employée utilement. Ce qu'un particulier ferait avec 60.000 livres, il faut ajouter un zéro quand il s'agit de Sa Majesté. Mais le mal devient plus terrible quand au lieu de bon pain et aussi des autres provisions que le Roi paye cher, le matelot et le soldat ne trouvent sous la dent qu'un amas de terre, de sable et de farine" ${ }^{2}$. Pour remonter jusqu'aux auteurs principaux de ces "voleries" comme on disait alors, il eut fallu faire une enquête dans les bureaux de la Marine. C'est ce que personne n'osait ni ne voulait faire. La perte au Canada fit éclater le scandale des malversations et des affaires de l'intendant Bigot. "C'est vous qui avez perdu le Canada", lui dit le ministre Berryer. Mais personne ne songea à accuser le premier commis La Porte qui avait couvert François Bigot de sa protection et qui était son associé. Arnaud de la Porte, lors du procès de Bigot, était confortablement à la retraite. Il était riche et honoré et le demeura ${ }^{53}$.

C'est que les Premiers Commis étaient de grands personnages. Ils avaient le titre de Conseillers du Roi en ses Conseils et le rang de sous-Secrétaires d'État ${ }^{54}$. A leur retraite, les Premiers Commis conser-

51. A. Duchêne, op. cit.

52. Observations sur les abus qui existent dans la Marine, 2 décembre 1750, (anonyme). AM., G. 121.

53. AM., C. 7. Dossier La Porte.

54. H. de Resbecq, op. cit. 
vaient souvent leurs appointements ${ }^{55}$. De plus, ils recevaient de royales gratifications. Lorsque Mlle Accaron, fille du premier commis en retraite, épousa en 1765 le capitaine de vaisseau comte de Grasse, Louis XV donna à la jeune fille une dot de 120.000 livres, ce qui était une fortune ${ }^{56}$.

Les historiens du Canada ont souvent accusé la monarchie française d'avoir négligé la colonie et finalement de l'avoir perdue. Ce pauvre Louis XV surtout a eu une très mauvaise presse. Mais nos historiens ont très mal connu et même pas du tout ce qu'était l'administration de la Nouvelle-France par les bureaux de la Marine. Garneau emploie l'expression "la métropole", parfois il dit "la royauté" 57 pour désigner le gouvernement de Versailles. Mais il ne semble pas avoir su que "la métropole" et la "royauté", c'étaient le plus ordinairement les commis. Le pouvoir du roi de France, monarque absolu, était, en effet, bien souvent celui d'un des Premiers Commis. Et au lieu de charger la mémoire du roi et celle des ministres, il est parfois plus juste de s'en prendre aux fonctionnaires. Car ce n'est pas toujours Pontchartrain, Maurepas ou Berryer qui ont mal conduit de Versailles les affaires du Canada, mais Fontanieu, Forcade ou Arnaud de La Porte. Et si Louis XV et Choiseul ont abandonné le Canada en 1763 c'est parce que Accaron n'a pas su les persuader de le garder ${ }^{58}$.

R. La Roque de Roguebrune

55. AM, C. 2. 55.

56. H. de Resbecq, op. cit.

57. Garneau, Histoire du Canada (2 vol., éd. Alcan, Paris, 1928), I: 233.

58. Les documents de la série Colonies C II cités dans cette étude, sont des pièces originales. Le Service des Archives du Canada à Paris a obtenu de M. le ministre de la France-d'Outre-Mer et de M. le Chef du Service des Archives des Colonies l'autorisation de faire microfilmer la série Colonies C II pour les Archives Publiques du Canada. 